\title{
Threatening to burn the house down
}

\author{
Narpinder Malhi, MD, and Immad Kiani, MD
}

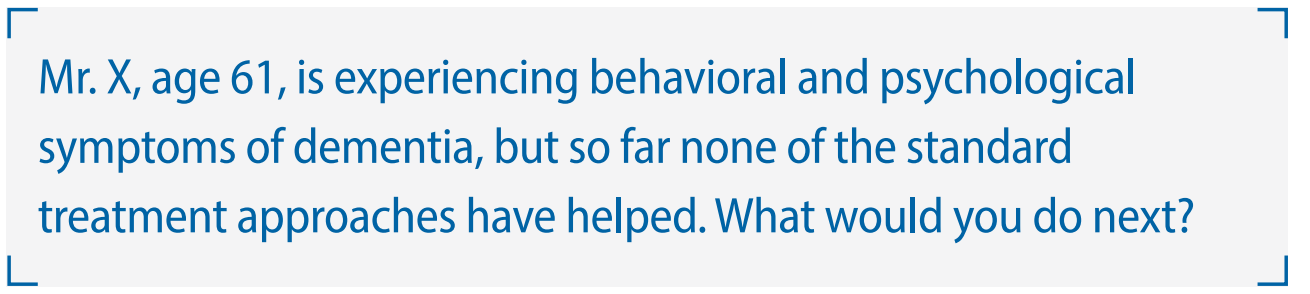

\section{CASE Agitated and aggressive}

Mr. X, age 61, who has Alzheimer's disease, is brought to the emergency department (ED) by his family after he is found to be confused, becomes physically aggressive with family members, and threatens to burn the house down. His family reports that earlier that day, he was paranoid that somebody was trying to kill him and he tried to leave the house. Mr. $X$ has been experiencing visual hallucinations and delusional thoughts that made him aggressive towards his son. After an initial laboratory workup in the ED, Mr. X's bloodwork comes back positive for mild leukocytosis, indicating the possibility of an infectious etiology. Mr. $X$ is admitted to the hospital for further evaluation of his altered mental status.

\section{HISTORY Decline over 2 years}

This is Mr. X's third inpatient admission for agitation and psychosis. His current medications-twice daily divalproex sodium extended release (ER), $250 \mathrm{mg}$ every morning and $500 \mathrm{mg}$ at every bedtime, and prazosin, $2 \mathrm{mg} / \mathrm{d}$ at bedtime-have been only partially effective. His medical history includes osteoarthritis, back pain, and heterozygous factor V Leiden (not on anticoagulation). He quit smoking tobacco several years ago and has no history of substance use. He has no family history of dementia. Previous trials of cholinesterase inhibitors, antipsychotics, and antidepressants resulted in only minimal improvement in his agitation and psychosis.

A chart review shows that 2 years before his current hospital admission, Mr. X had presented to his primary care physician with slurred speech, forgetfulness, missing words, and transient reading difficulties. His initial laboratory workup and MRI came back normal. He was placed on short-term disability due to work-related errors. He was referred to the hospital's Memory Clinic 2 years ago, where his Mini-Mental State Exam score was 20/30, indicating mild cognitive impairment. Stroke workup was negative. Due to significant language deficits, a differential diagnosis for Alzheimer's disease vs primary progressive aphasia vs frontotemporal dementia was made. He screened positive for amyloid PET scan, which confirmed the diagnosis of Alzheimer's disease.

Neuropsychological testing showed similarities with logopenic variant of primary progressive aphasia, which in many cases is present in Alzheimer's disease. Mr. X was

Dr. Malhi is Child and Adolescent Psychiatrist Faculty, Department of Psychiatry, ChristianaCare Health System, Newark, Delaware. Dr. Kiani is a PGY-3 Psychiatry Resident, Department of Psychiatry, ChristianaCare Health System, Newark, Delaware.

Disclosures

The authors report no financial relationships with any companies whose products are mentioned in this article, or with manufacturers of competing products.

doi: $10.12788 /$ cp.0077

\section{How would you handle this case?}

Answer the challenge questions at MDedge.com/ psychiatry and see how your colleagues responded 


\section{Clinical Point}

\section{Nonpharmacologic interventions are the} first-line treatment for patients with BPSD

Discuss this article at www.facebook.com/ MDedgePsychiatry prescribed anticholinesterase inhibitors, including donepezil, $10 \mathrm{mg} / \mathrm{d}$, and rivastigmine patch, $9.5 \mathrm{mg} / \mathrm{d}$; and memantine, 10 $\mathrm{mg} / \mathrm{d}$, which he could not tolerate because of adverse effects. During the next year, Mr. $X$ deteriorated and presented to the ED a few times with significant psychotic symptoms and aggression. He had a poor response to various pharmacologic and nonpharmacologic interventions during this time.

\section{EVALUATION Continued \\ problematic behaviors}

During his hospitalization, Mr. $\mathrm{X}$ continues to be agitated and paranoid and is placed in restraints. He is unable to respond to his name and cannot follow simple verbal commands. Results of his laboratory workup are within normal limits. His mild leukocytosis resolves with no active signs of infection. Psychiatry is consulted for management of his behavioral and psychological symptoms of dementia (BPSD).

Mr. $\mathrm{X}$ is started on olanzapine and lorazepam as needed for agitation, and his twice daily divalproex sodium ER is increased to 250 every morning and $750 \mathrm{mg}$ at every bedtime. However, Mr. X remains agitated and requires restraints. Olanzapine is switched from an as-needed dose to scheduled doses of $10 \mathrm{mg}$ every morning and $15 \mathrm{mg}$ at every bedtime, to address his psychosis and agitation.

On Day 24 of hospitalization, Mr. X's ammonia levels are checked and are found to be $69 \mu / \mathrm{dL}$, which is high (normal range: 15 to $45 \mu / \mathrm{dL}$ ). Divalproex sodium ER is eventually tapered and discontinued. Mr. $X$ is started on carbamazepine, which is titrated to $400 \mathrm{mg}$ twice daily and results in some improvement in his behavior. He continues to receive carbamazepine and is started on dextromethorphan-quinidine, $10 \mathrm{mg} / \mathrm{d}$, and increased to $10 \mathrm{mg}$ twice daily; however, Mr. X continues to be verbally aggressive with staff, throws food, wanders around, and tries to leave the hospital unit, so he is placed in restraints and continues to require a sitter.
What treatment approach would you consider next?

a) nonpharmacologic approaches such as behavioral therapy

b) pharmacologic treatment with a different antipsychotic

c) electroconvulsive therapy

\section{The author's observations}

Dementia typically affects older adults, but its onset can occur before age 60 . It is a syndrome rather than a specific illness; the most common types are Alzheimer's disease, vascular dementia, dementia with Lewy bodies, and frontotemporal dementia. Diagnostic clarity and an evidence-based treatment plan are crucial for improving the quality of life for both the patient and their caregivers. The Table (page 41) outlines the differential diagnosis of cognitive deficits. New-onset cognitive deficits warrant neuroimaging, and other testing may also be needed.

\section{Behavioral and psychological symptoms of dementia}

Noncognitive symptoms occur in $98 \%$ of individuals with dementia at some point in their disease and are often the most distressing to both caregivers and patients. ${ }^{1}$ Behavioral and psychological symptoms of dementia, including apathy, depression, sleep disorders, hallucinations, delusions, psychosis, agitation, and aggression, are exceedingly prevalent. ${ }^{2}$ Although these symptoms pose a significant burden, there are no clear published treatment guidelines; however, the American Psychiatric Association and the American Geriatric Society recommend using nonpharmacologic approaches as the first-line of treatment for patients with BPSD.,4

\section{Nonpharmacologic treatments}

Due to the unfavorable adverse effects profiles of medications commonly used to treat dementia, nonpharmacologic treatment 


\section{Differential diagnosis of cognitive deficits}

\begin{tabular}{lll} 
Disease/condition & Hallmark features \\
\hline Alzheimer's disease & $\begin{array}{l}\text { Cognitive decline, language deficits, apraxia, agnosia, and eventual } \\
\text { functional decline }\end{array}$ \\
\hline Frontotemporal dementia & $\begin{array}{l}\text { Changes in personality and behaviors, involves temporal and frontal } \\
\text { lobes, early language difficulties }\end{array}$ \\
\hline Vascular dementia & History of cerebrovascular accidents, stepwise decline \\
\hline Dementia with Lewy bodies & Visual hallucinations and rigidity (parkinsonism features), sensitivity \\
& to antipsychotics
\end{tabular}

approaches have always played a crucial role for managing BPSD. Interventions such as music therapy, aromatherapy, art therapy, behavioral therapy, reality orientation, tailored activities, and physical exercises, have shown promising results for alleviating BPSD. ${ }^{5-7}$

\section{Pharmacologic treatments}

Pharmacologic therapies should be used when nonpharmacologic approaches are unsuccessful, or when a patient is at imminent risk to harm themselves or others.

Antipsychotics. Although there is conflicting data regarding the use of antipsychotics in older adults, these agents are the most common pharmacologic treatment for patients with BPSD. Several studies examining the efficacy of antipsychotics for treating BPSD have demonstrated an increased risk of cerebrovascular events, including stroke and death due to any cause. ${ }^{8}$ While the use of antipsychotics increases the risk of mortality in older adults, the absolute risk is still low. ${ }^{9}$

Antipsychotics used to treat BPSD include:

- Risperidone is well studied in older adults and has shown benefit for treating aggression, agitation, and psychosis. ${ }^{10}$

- Quetiapine has a favorable adverse effects profile and may help improve sleep and reduce anxiety. ${ }^{10}$

- Olanzapine. Low-dose olanzapine has been modestly effective in decreasing agitation and aggression in patients with Alzheimer's and vascular dementias. ${ }^{11}$

- Aripiprazole has shown modest benefit in treating psychosis and agitation in patients with dementia but may be associated with insomnia or activation symptoms at lower doses. ${ }^{10}$

- Ziprasidone. Case reports have found benefit with oral and injectable forms. ${ }^{12}$

Antidepressants. In the CitAD study, which was a placebo-controlled randomized trial,

\section{Clinical Point \\ Pharmacologic therapies for patients with BPSD should be used when nonpharmacologic approaches are unsuccessful}




\section{Clinical Point}

ECT may be an option

\section{for patients with}

BPSD who do not

respond to multiple

pharmacologic

treatments

\section{Related Resources}

- Van den Berg JF, Kruithof HC, Kok RM, et al. Electroconvulsive therapy for agitation and aggression in dementia: a systematic review. Am J Geriatr Psychiatry. 2018;26(4):419-434.

- Kales HC, Mulsant BH, Sajatovic M. Prescribing antipsychotics in geriatric patients: Focus on dementia. Current Psychiatry. 2017;16(12):24-30.

\section{Drug Brand Names}

Aripiprazole • Abilify

Carbamazepine - Tegretol

Citalopram - Celexa

Dextromethorphan-

quinidine - Nuedexta

Divalproex sodium

ER-Depakote

Donepezil • Aricept

Gabapentin - Neurontin

Haloperidol $\cdot$ Haldol

citalopram titrated to a target of $30 \mathrm{mg} / \mathrm{d}$ was found to be effective in reducing BPSD. ${ }^{13}$ However, QTc prolongation limits the use of citalopram. Sertraline was studied in 1 small, randomized trial against haloperidol but showed no additional benefit. ${ }^{14}$

Mood stabilizers. In a small, randomized trial, carbamazepine was helpful for patients with BPSD who were resistant to treatment with antipsychotics, with efficacy demonstrated over 6 weeks. ${ }^{15}$ No other mood stabilizers have had significant positive results in treating BPSD. ${ }^{16}$

Anxiolytic medications. Some research suggests that the occasional use of lorazepam, as necessary, is acceptable for patients with extreme agitation or aggression when behavioral interventions or sleep aids are ineffective. ${ }^{17}$ Various case reports and case series have suggested gabapentin may be effective for BPSD. ${ }^{18}$

Prazosin. In a small randomized placebo-controlled trial, the commonly used antihypertensive agent prazosin reduced agitation and aggression in patients with Alzheimer's dementia, at doses from 1 to $6 \mathrm{mg} / \mathrm{d} .{ }^{19}$ Postural hypotension, the main adverse effect associated with prazosin, can limit its use.

Trazodone. Some research suggests trazodone can reduce irritability and aggression in patients with Alzheimer's disease..$^{20}$

Dextromethorphan/quinidine. In a 10-week phase 2 randomized clinical trial of patients with probable Alzheimer's disease dementia, combination dextromethorphan/quinidine reduced agitation and was generally well tolerated. ${ }^{21}$

For patients such as $\mathrm{Mr}$. X who do not respond to multiple pharmacologic treatments, electroconvulsive therapy (ECT) may be an option.

\section{TREATMENT A trial of ECT}

Because Mr. X does not respond to the standard treatment protocols, the treatment team and Mr. X's family discuss the use of ECT to control his agitation. Consent is obtained from his legal guardian and Mr. $X$ is medically cleared to receive ECT. Mr. $X$ receives $3 \mathrm{ECT}$ treatments per week. During the first week, Mr. X experiences post-treatment agitation and confusion. The frequency of ECT treatments is reduced to 2 treatments per week, and then 1 session per week. Mr. X starts to

\section{Bottom Line}

Behavioral and psychological symptoms of dementia are associated with poor outcomes and a greater burden of care. Nonpharmacologic interventions should be the first-line treatment. The risk of prescribing medications for older adults should be carefully weighed against possible benefits, and pharmacotherapy should be combined with nonpharmacologic options for optimum results. Electroconvulsive therapy can be considered when standard treatment options are not successful. 
show improvement in his agitation and ECT is continued at 1 session per week for 7 weeks.

\section{The authors' observations}

Electroconvulsive therapy has been an effective treatment for patients with treatmentresistant depression and has shown benefit in treating other psychiatric conditions such as acute mania, catatonia, psychotic disorders, and Parkinson's disease. ${ }^{22}$ Its use as an offlabel treatment for chronic neuropathic pain has also been well documented. ${ }^{23}$ Although ECT is not indicated for treating agitation and aggression in patients with dementia, its effectiveness for these symptoms has been discussed extensively in the literature. 22,2426

Electroconvulsive therapy treatment can be divided into 2 phases: an acute phase during which ECT is administered 2 to 3 times a week for 4 to 5 weeks, and a maintenance phase of weekly treatments for 4 weeks and then biweekly treatments for 8 weeks. ${ }^{26}$ Although extensive research supports the safe use of ECT in older adults, concerns for worsening cognitive impairment can deter patients and families from agreeing to this treatment.

Adverse effects of ECT such as headaches and postictal confusion are generally mild and transient. Severe adverse effects such as seizures, severe confusion, and delirium are uncommon. ${ }^{25}$ The number of ECT treatments required for a good effect ranges from 2 to 18 , and the most common position for electrodes placement is bilateral. Outcomes can be measured by using rating scales such as the Cohen-Mansfield Agitation Inventory, Neuropsychiatric Inventory, Social Dysfunction and Aggression Scale, Clinical Global Impression scale, and Pittsford Agitation Scale. ${ }^{25}$ Obtaining consent from patients with dementia is generally not possible because these patients generally lack the capacity to make medical decisions. Clinicians should refer to their state laws regarding medical-decision making in such cases. The patient's next of kin or medical power of attorney should be contacted, and the risks and benefits should be discussed before starting ECT.

\section{OUTCOME Lasting improvement}

Due to Mr. X's improvement after ECT, on hospital Day 124, the restraints are removed and he no longer requires a sitter. He starts responding to his name and following simple verbal commands. Electroconvulsive therapy is tapered to every other week, and eventually stopped as his status improves. Mr. $\mathrm{X}$ continues to do well and is maintained on the same dosages of olanzapine, carbamazepine, and dextromethorphan-quinidine he had been receiving prior to discharge.

\section{References}

1. Kales HC, Gitlin LN, Lyketsos CG. Management of neuropsychiatric symptoms of dementia in clinical settings: recommendations from a multidisciplinary expert panel. J Am Geriatr Soc. 2014;62(4):762-769.

2. Scarmeas N, Brandt J, Albert M, et al. Delusions and hallucinations are associated with worse outcome in Alzheimer disease. Arch Neurol. 2005;62(10):1601-1608.

3. Reus VI, Fochtmann LJ, Eyler AE, et al. The American Psychiatric Association Practice Guideline on the use of antipsychotics to treat agitation or psychosis in patients with dementia. Am J Psychiatry. 2016;173(5):543-546.

4. AGS Executive Committee. A guide to the management of psychotic disorders and neuropsychiatric symptoms of dementia in older adults. The American Geriatrics Society. Published April 2011. Accessed September 24, 2020. https:// qioprogram.org/sites/default/files/AGS_Guidelines_for_ Telligen.pdf

5. Yang MH, Lin LC, Wu SC, et al. Comparison of the efficacy of aroma-acupressure and aromatherapy for the treatment of dementia-associated agitation. BMC Complement Altern Med. 2015;15:93.

6. Cerga-Pashoja A, Lowery D, Bhattacharya R, et al Evaluation of exercise on individuals with dementia and their carers: a randomised controlled trial. Trials. 2010;11:53.

7. Chen RC, Liu CL, Lin MH, et al. Non-pharmacological treatment reducing not only behavioral symptoms, but also psychotic symptoms of older adults with dementia: a prospective cohort study in Taiwan. Geriatr Gerontol Int. 2014;14(2):440-446.

8. Schneider LS, Dagerman KS, Insel P. Risk of death with atypical antipsychotic drug treatment for dementia: metaanalysis of randomized placebo-controlled trials. JAMA. 2005;294(15):1934-1943.

9. Lenzer J. FDA warns about using antipsychotic drugs for dementia. BMJ. 2005;330(7497):922.

10. Burke AD, Tariot PN. Atypical antipsychotics in the elderly: a review of therapeutic trends and clinical outcomes. Expert Opin Pharmacother. 2009;10(15):2407-2414.

11. Moretti R, Torre R, Antonello T, et al. Olanzapine as a possible treatment of behavioral symptoms in vascular dementia: risks of cerebrovascular events. J Neurol. 2005;252(10): 1186-1193.

12. Cole SA, Saleem R, Shea WP, et al. Ziprasidone for agitation or psychosis in dementia: four cases. Int J Psychiatry Med 2005;35(1):91-98.

13. Porsteinsson AP, Drye LT, Pollock BG, et al. Effect of citalopram on agitation in Alzheimer disease: the CitAD randomized clinical trial. JAMA. 2014;311(7):682-691.

\section{Clinical Point}

ECT is not indicated for treating agitation and aggression in patients with dementia, but some evidence supports its use in such cases 


\section{Clinical Point}

\section{Although research supports the safe use of ECT in older adults, concerns for worsening cognitive impairment can deter its use}

14. Gaber S, Ronzoli S, Bruno A, et al. Sertraline versus small doses of haloperidol in the treatment of agitated behavior in patients with dementia. Arch Gerontol Geriatr Suppl. 2001; 7:159-162.

15. Olin JT, Fox LS, Pawluczyk S, et al. A pilot randomized trial of carbamazepine for behavioral symptoms in treatmentresistant outpatients with Alzheimer disease. Am J Geriatr Psychiatry. 2001;9(4):400-405.

16. Konovalov S, Muralee S, Tampi RR. Anticonvulsants for the treatment of behavioral and psychological symptoms of dementia: a literature review. Int Psychogeriatr. 2008;20(2):293-308.

17. Davies SJC, Burhan AM, Kim D. Sequential drug treatment algorithm for agitation and aggression in Alzheimer's and mixed dementia. J Psychopharmacol. 2018;32(5):509-523.

18. Kim Y, Wilkins KM, Tampi RR. Use of gabapentin in the treatment of behavioural and psychological symptoms of dementia: a review of the evidence. Drugs Aging. 2008;25(3):187-196.

19. Wang LY, Shofer JB, Rohde K, et al. Prazosin for the treatmen of behavioral symptoms in patients with Alzheimer disease with agitation and aggression. Am J Geriatr Psychiatry. 2009; 17(9):744-751.

20. López-Pousa S, Garre-Olmo J, Vilalta-Franch J, et al.
Trazodone for Alzheimer's disease: a naturalistic follow-up study. Arch Gerontol Geriatr. 2008;47(2):207-215.

21. Cummings JL, Lyketsos CG, Peskind ER. Effect of dextromethorphan-quinidine on agitation in patients with Alzheimer disease dementia: a randomized clinical trial. JAMA. 2015;314(12):1242-1254.

22. Ujkaj M, Davidoff DA, Seiner SJ, et al. Safety and efficacy of electroconvulsive therapy for the treatment of agitation and aggression in patients with dementia. Am J Geriatr Psychiatry. 2012;20(1):61-72.

23. McDaniel WW. Electroconvulsive therapy in complex regional pain syndromes. J ECT. 2003;19(4):226-229.

24. Glass OM, Forester BP, Hermida AP. Electroconvulsive therapy (ECT) for treating agitation in dementia (major neurocognitive disorder)-a promising option. Int Psychogeriatr. 2017;29(5):717-726.

25. Van den Berg JF, Kruithof HC, Kok RM, et al. Electroconvulsive therapy for agitation and aggression in dementia: a systematic review. Am J Geriatr Psychiatry. 2018;26(4):419-434.

26. Isserles M, Daskalakis ZJ, Kumar S, et al. Clinical effectiveness and tolerability of electroconvulsive therapy in patients with neuropsychiatric symptoms of dementia. J Alzheimers Dis. 2017;57(1):45-51. 